\title{
Mid-wave InAs/GaSb Superlattice PiBN Infrared Photodetector Grown on GaAs Substrate
}

\author{
Jian Huang \\ School of Information Science and \\ Technology \\ ShanghaiTech University \\ Shanghai China 201210 \\ huangjian@shanghaitech.edu.cn \\ Huiyun Liu \\ Department of Electronic and Electrical \\ Engineering \\ University College London \\ London United Kingdom \\ huiyun.liu@ucl.ac.uk
}

\author{
Daqian Guo \\ Department of Electronic and Electrical \\ Engineering \\ University College London \\ London United Kingdom \\ daqian.guo.15@ucl.ac.uk \\ Jiang Wu \\ Department of Electronic and Electrical \\ Engineering \\ University College London \\ London United Kingdom \\ jiang.wu@ucl.ac.uk
}

\author{
Zhuo Deng \\ School of Information Science and \\ Technology \\ ShanghaiTech University \\ Shanghai China 201210 \\ dengzhuo@shanghaitech.edu.cn; \\ Baile Chen \\ School of Information Science and \\ Technology \\ ShanghaiTech University \\ Shanghai China 201210 \\ chenbl@shanghaitech.edu.cn
}

\begin{abstract}
We present a GaAs based PiBN InAs/GaSb type II superlattice photodetector for mid infrared application with peak responsivity of $0.44 \mathrm{~A} / \mathrm{W}$ at $4.1 \mathrm{um}$ at $77 \mathrm{~K}$ under $0 \mathrm{~V}$.
\end{abstract}

Keywords-InAs/GaSb type-II superlattices, infrared detectors, barrier detectors, mid wavelength

\section{INTRODUCTION}

Recently, InAs/GaSb type II superlattice(T2SL) has attracted lots of interests in infrared photodetectors due to its tunable operating wavelength, which can be toiled form 3 um to $32 \mathrm{um}$ by adjusting the thickness of InAs or GaSb layer [1] In addition, T2SL materials also have some advantages than traditional $\mathrm{HgCdTe}$, such as good uniformity, lower leakage current and larger effective mass which can reduce tunneling current. Incorporating a barrier in a detector can eliminate the generation-recombination (GR) currents associated with Shockley-Read-Hall (SRH) centers and mesa lateral surface imperfections, which have resulted in an increase of operating temperature [2]. The PiBN is a unipolar barrier blocks one carrier type (electrons) but allows unimpeded flow of the other (holes). The introduction of a unipolar barrier into various configurations of photovoltaic structures suppresses dark current and noises without impending photocurrent flow.

Currently, the high cost and small size of commercially available GaSb substrates is a huge obstacle to large volume production. In this paper, we present a PiBN InAs/GaSb T2SL photodetector grown on GaAs substrate, can could be more attractive for high volume, high-throughput manufacture.

\section{DEVICE DESIGN}

Fig. 1 shows the structure of the device. The devices were grown on GaAs substrate, following the substrate was the buffer layer. Then a $500 \mathrm{~nm}$ thick p type bottom contact layer was formed by 10 monolayer InAs(ML) /10 ML GaSb SLs doped with Be, followed by a $2000 \mathrm{~nm}$ thick nonintentionally doped (n.i.d.) absorber region formed by $10 \mathrm{ML}$ InAs/10 ML GaSb SLs. Then a $250 \mathrm{~nm}$ thick $6 \mathrm{ML}$ AlSb/12 ML InAs SLs barrier was grown, followed by a $200 \mathrm{~nm}$ thick $\mathrm{n}$ type top contact layer formed by $5 \mathrm{InAs}(\mathrm{ML}) / 5 \mathrm{ML} \mathrm{GaSb}$ $\mathrm{SLs}$ doped with $\mathrm{Si}$. The contact layer was capped with a 50 $\mathrm{nm}$ thick $\mathrm{n}$ InAs to improve ohmic contact properties.
After the material growth, the wafer was processed into a set of mesa-isolated test structures with device diameters ranging from $20 \mu \mathrm{m}$ to $500 \mu \mathrm{m}$ using standard UV photolithographic processing technique. Mesa structures were defined by citric-acid based wet etching and mesa slopes were passivated by SU- 8 . Finally, $50 \mathrm{~nm} / 50 \mathrm{~nm} / 300 \mathrm{~nm}$ $\mathrm{Ti} / \mathrm{Pt} / \mathrm{Au}$ were deposited by e-beam evaporator as the top and bottom contact layers.

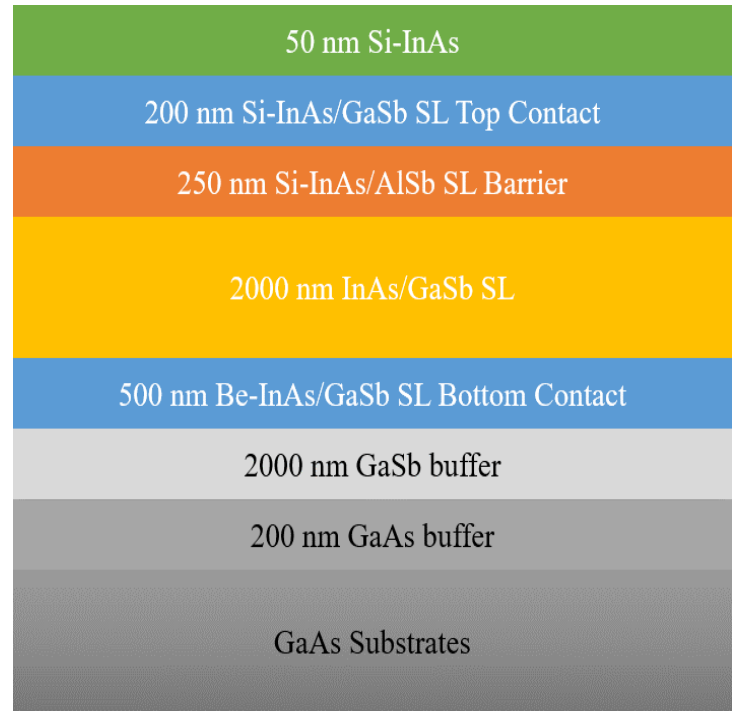

Fig. 1 Schematic of the device structure.

\section{RESULTS}

Fig. 2 shows the dark current of a 130um device based on GaAs from $77 \mathrm{~K}$ to $300 \mathrm{~K}$ which were measured in low temperature probe station by semiconductor device analyzer. Dark current measured at $77 \mathrm{~K}$ shows a small photovoltaic shift, which is due to the imperfections in the cold shield used for the measurement. Fig. 3 shows the calculated resistance area product (ROA) as a function of temperature, from this Arrhenius plot the activation energy $\left(E_{a}\right)$ can be estimated. The linear fits yield an activation energy of 98 $\mathrm{meV}$ at high temperature region, which indicates the dark current is dominated by generation-recombination component. 
The optical response was measured by Fourier transform infrared spectrometer and calibrated by a blackbody resource at $700^{\circ} \mathrm{C}$ under three different bias as shown in Fig. 4. The device shows the peak responsivity is $\sim 0.5 \mathrm{~A} / \mathrm{W}$ at $4.1 \mu \mathrm{m}$ at $77 \mathrm{~K}$ under $-0.1 \mathrm{~V}$, the $50 \% \lambda \mathrm{c}$ is $\sim 6.1 \mu \mathrm{m}$ and the $100 \% \lambda \mathrm{c}$ is $\sim 6.6 \mu \mathrm{m}$.

The specific detectivity $\mathrm{D}^{*}$ was estimated using:

$$
D^{*}=\frac{R_{\lambda} \sqrt{A}}{\left(2 q I+\frac{4 \mathrm{k}_{B} \mathrm{~T}}{R}\right)^{1 / 2}}
$$

where $\mathrm{q}$ is the electronic charge, $\mathrm{k}_{\mathrm{B}}$ is Boltzmann's constant, $\mathrm{T}$ is the temperature of the device, $\mathrm{R}$ is the resistance under the bias, $\mathrm{R}_{\lambda}$ is the responsivity, $\mathrm{I}$ is dark current and $\mathrm{A}$ is the area of device. A peak detectivity $\mathrm{D}^{*}$ of $1.3 \times 10^{11} \mathrm{cmHz}^{1 / 2} / \mathrm{W}$ was calculated at $4.1 \mu \mathrm{m}$ at $77 \mathrm{~K}$ under $0 \mathrm{~V}$ as shown in Fig. 5.

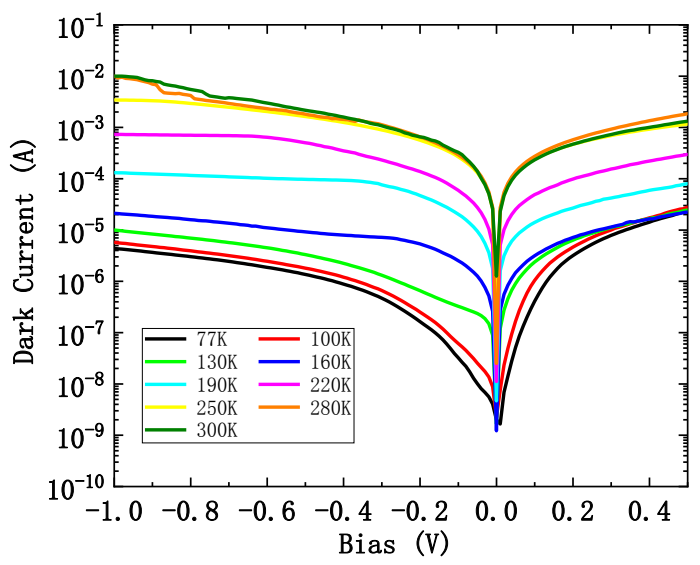

Fig 2. Dark current of the $130 \mu \mathrm{m}$ diameter GaAs based PiBN device as a function of temperature.

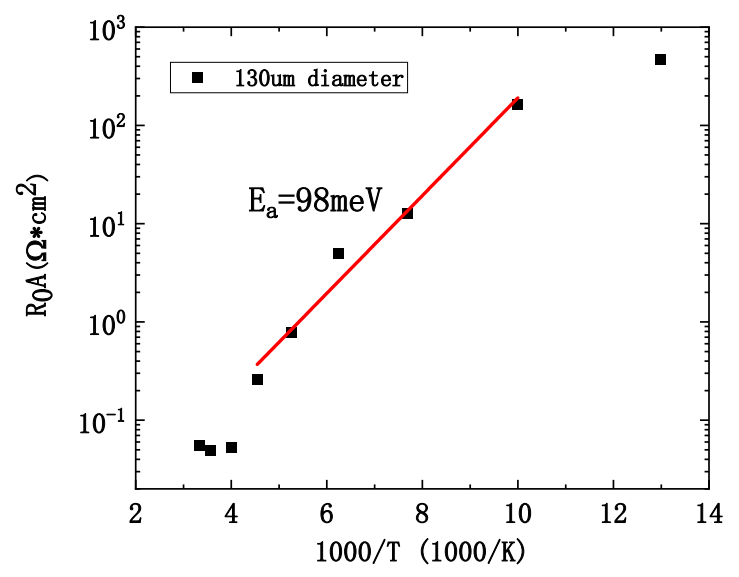

Fig .3 Arrhenius plot of the $\mathrm{R}_{0} \mathrm{~A}$ product

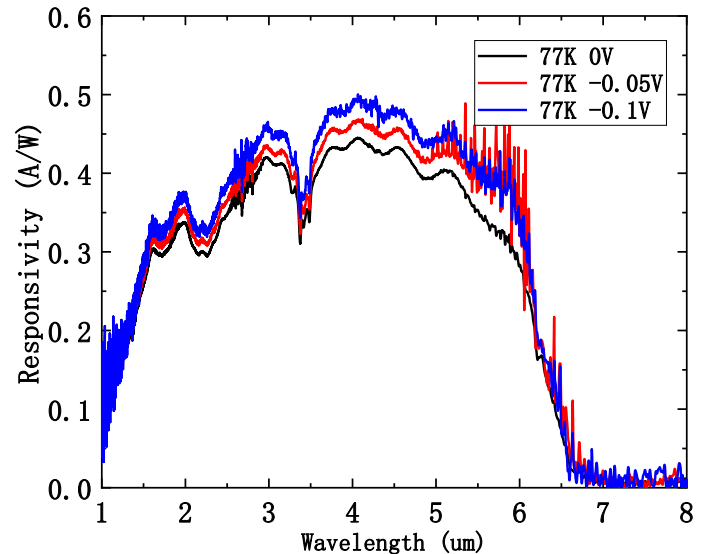

Fig .4 Optical response of detector measured at $77 \mathrm{~K}$ under reverse bias of $0 \mathrm{~V},-0.05 \mathrm{~V}$ and $-0.1 \mathrm{~V}$.

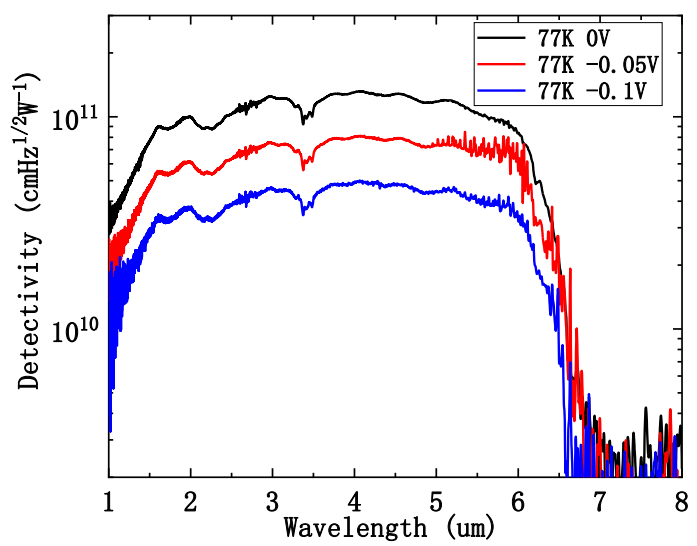

Fig.5 The specific detectivity of the device at $77 \mathrm{~K}$ under reverse bias of $0 \mathrm{~V}$, $-0.05 \mathrm{~V}$ and $-0.1 \mathrm{~V}$.

\section{CONCLUSION}

In summary, we demonstrated a PiBN InAs/GaSb T2SL photodetector grown on GaAs substrate. The device has a peak responsivity of $0.44 \mathrm{~A} / \mathrm{W}$ and a peak specific detectivity of $1.3 \times 10^{11} \mathrm{cmHz}^{1 / 2} / \mathrm{W}$ at $4.1 \mathrm{um}$ at $77 \mathrm{~K}$ under $0 \mathrm{~V}$.

\section{ACKNOWLEDGMENT}

This work was supported by Shanghai Sailing Program(17YF1429300), ShanghaiTech University startup funding (F-0203-16-002), and UK EPSRC First Grant (No. $\mathrm{EP} / \mathrm{R} 006172 / 1)$.

\section{REFERENCES}

[1] E. A. Plis, "InAs/GaSb Type-II Superlattice Detectors," Advances in Electronics, vol. 2014, pp. 1-12, 2014.

[2] B. F. Andresen, P. Klipstein, G. F. Fulop, and P. R. Norton, ""XB n " barrier photodetectors for high sensitivity and high operating temperature infrared sensors," Proc. of SPIE 2008. 